\title{
El Juez de Vigilancia Penitenciaria: Un modelo para la creación de juzgados de reinserción en las jurisdicciones angloamericanas en aplicación de los principios del "derecho terapéutico"
}

\author{
David B Wexler ${ }^{*}$ y Jeanine Calderón ${ }^{* *}$
}

\begin{abstract}
RESUMEN
La estrecha relación que existe entre la rehabilitación y el éxito de la reinserción social ha generado un especial interés en esta última en tiempos recientes. El aparente éxito que han tenido los juzgados de tratamiento contra la drogadicción ha generado una serie de propuestas para la creación de juzgados de reinserción social en los que los jueces supervisen de forma coordinada el progreso del recluso, el cumplimiento de las condiciones para su puesta en libertad provisional y su reincorporación a la sociedad. Dado que en los sistemas angloamericanas la función del juez termina normalmente una vez que dicta la sentencia, sería necesario adoptar un nuevo modelo que permitiera el establecimiento y funcionamiento de juzgados de reinserción. En cambio, España cuenta en la actualidad con una figura judicial, el Juez de Vigilancia Penitenciaria (JVP), que mantiene jurisdicción sobre el reo durante el cumplimiento de la condena. En este artículo expondremos como la figura del JVP podría servir como de modelo jurídico para el establecimiento de juzgados de reinserción en los sistemas angloamericanos.
\end{abstract}

\begin{abstract}
Prisoner 're-entry' into society is a major topic of current concern. Some commentators urge breathing new life into the parole process. The apparent success of drug treatment courts and other problem-solving courts has led to a different suggestion: a re-entry court, whereby a judge would oversee and coordinate the prisoner's progress, conditional release, and reintegration. In Anglo-American jurisdictions, judicial authority typically ends with the imposition of an incarcerative sentence, and thus we are very much in need of a structural model to use in constructing a re-entry court. In Spain, there is a judge (the juez de vigilancia penitenciaria or JVP) designated to oversee the correctional component, including the authority to grant and revoke conditional release. This article discusses how such a legal structure could be used as a model for the legal structure needed in Anglo-Americans jurisdictions in order to construct a re-entry court.
\end{abstract}

\section{Introducción}

En los Estados Unidos, la puesta en libertad y reinserción social de los presos es una preocupación de primer orden. En el sistema de discretionary parole vigente hasta hace poco en el país, la decisión sobre la concesión de la libertad condicional residía en órganos administrativos colegiados que gozaban de amplia discreción para la puesta en libertad de los convictos. Sin embargo, a raíz de una serie de reformas recientes, tendentes a promover sistemas fijos o reglados de determinación de la pena, se ha asistido a la práctica desaparición del sistema de parole en la mayoría de las jurisdicciones del país. Estas reformas han tenido efectos positivos, especialmente desde la perspectiva de

\footnotetext{
* Lyons Professor of Law, Profesor de Psicología en la Universidad de Arizona y Profesor de Derecho en la Facultad de Derecho de la Universidad de Puerto Rico. Agradecemos los comentarios y sugerencias de la Prof. Cristina Urios, el Prof. Marco Aparicio y el Dr. Luis Rodríguez-Piñero Se puede contactar con el autor en la siguiente direccion electronica: davidbwexler@yahoo.com

${ }^{* *}$ Estudiante de Derecho en la Universidad de Puerto Rico.
}

Revista Española de Investigación Criminólogica REIC AC-01-04 http://www.criminologia.net

ISSN 1696-9219

https://doi.org/10.46381/reic.v2i0.14 
la igualdad de trato, pero también ha introducido muchos problemas en términos de la integración de los presos en la sociedad.

En este sentido, algunos comentaristas han abogado por el restablecimiento de un sistema de libertad condicional, aunque sujeto a nuevas garantías y circunscrito a una discrecionalidad limitada (Petersilia, 2003). Otra propuesta interesante es el establecimiento de tribunales de reinserción social (reentry courts), a través de las cuales el poder judicial autorizaría, supervisaría y, de ser necesario, revocaría la libertad condicional. Esta propuesta de involucrar al poder judicial en el proceso de reinserción social resulta particularmente novedosa desde la perpectiva de los sistemas jurídicos angloamericanos, donde el papel del juez acaba con la imposición de una pena privativa de libertad. A pesar de ello, los jueces de estos sistemas cuentan con una rica experiencia en el trabajo con los delicuentes en contextos anteriores al encarcelamiento, como es el caso de los tribunales para el tratamiento de la drogadicción, los tribunales para la violencia doméstica y otros tipos de tribunales especializados en la "resolución de problemas" (Winick and Wexcler, 2003), y se ha sugerido que el tipo de relación que se crea entre el delincuente y el juez en este tipo de contextos puede servir también para motivar la rehabilitación de éstos tras cumplir una pena privativa de libertad. Es en esta misma dirección donde apunta la propuesta de dotar al sistema jurídico de los Estados Unidos de un nuevo marco jurídico para la creación de tribunales de reinserción, donde los jueces puedan trabajar directamente con el delincuente para promover su reinserción a la sociedad.

España cuenta ya con un marco normativo en el que el Juez de Vigilancia Penitenciaria (JVP) desempeña muchas de las funciones que se están proponiendo ahora en los Estados Unidos en relación con los posibles tribunales de reinserción. En este artículo, recurrimos al marco jurídico que regula la figura del JVP para hacer propuestas en torno a la creación de tribunales de reinserción en los sistemas angloamericanos. Para ello, combinaremos nuestro análisis de la legislación española con los principios del denominado "derecho terapéutico" (therapeutic jurisprudence) ${ }^{1}$, una teoría que reconoce el importante potencial terapéutico de los distintos actores jurídicos.

La therapeutic jurisprudence presta atención a ciertas áreas, anteriormente no tomadas en consideración, con el objetivo de humanizar el derecho, atendiendo a las facetas humanas, psicológicas y emocionales de las normas y procesos jurídicos (Winick \& Wexler, 2003). Para lograr su objetivo, el derecho terapéutico integra aspectos de la psicología, criminología y otras ciencias del comportamiento humano, integradas en una perspectiva interdisciplinar. En principio, hay que reconocer que tanto la aplicación de las normas jurídicas como los procesos jurídicos producen un impacto en las personas, y un impacto que puede ser tanto positivo o negativo, es decir, terapéutico o

\footnotetext{
${ }^{1}$ Ha resultado difícil conseguir una traducción del término con la cual se pueda mantener el significado completo de "therapeutic jurisprudence". Una traducción literal al castellano sería "jurisprudencia terapéutica" pero en inglés la palabra "jurisprudence" tiene un significado más amplio que en castellano, y se refiere, en términos generales, a la teoría o filosofía jurídica. Otras traducciones del término que han sido empleadas con anterioridad son: "terapia jurídica," "justicia terapéutica," o "teoría del derecho terapéutico." Ninguna de estas expresiones abarca suficientemente la amplitud del contenido del término en inglés, por lo que ha preferido conservar ésta. "Therapeutic jurisprudence" es además el término de uso internacional de esta naciente disciplina.
}

Revista Española de Investigación Criminólogica

REIC AC-01-04

http://www.criminologia.net

ISSN 1696-9219 
antiterapéutico $^{2}$. La therapeutic jurisprudence nos emplaza a que seamos sensibles a estos aspectos, buscando vías para modificar las normas y procesos jurídicos de tal forma que produzcan efectos rehabilitadores y terapéuticos, salvaguardando siempre otros objetivos del sistema legal tales como la justicia y el debido proceso de la ley (Winick \& Wexler, 2003).

Aunque la therapeutic jurisprudence nació originalmente en el ámbito académico, su buena acogida y su rápido desarrollo han propiciado su consolidación en la práctica de los actores jurídicos, convirtiéndose en un instrumento efectivo para la promoción de cambio en los Estados Unidos y otros países del ámbito anglosajón, como Canadá, Australia, Nueva Zelanda, Escocia, etc. (Winick \& Wexler, 2003). Cuando los principios de la therapeutic jurisprudence se llevan a la práctica, nos encontramos con lo que se denomina un agente terapéutico. Un agente terapéutico puede ser tanto un abogado como el mismo juez, actores cuyo papel en el proceso jurídico puede convertirlos en piezas claves en la promoción del bienestar de las personas.

En los Estados Unidos, el caso más notable de aplicación de los principios de la therapeutic jurisprudence es el de los juzgados especializados de tratamiento, especialmente aquellos dedicados al tratamiento de adictos al alcohol y a otras sustancias psicotrópicas, o al control de personas responsables de actos de violencia doméstica (Winick \& Wexler, 2003). Dentro de estos juzgados, los más conocidos sean seguramente los especializados en el tratamiento de la drogadicción, que ofrecen a los drogadictos no resposanbles de actos de violencia la oportunidad de rehabilitarse mediante tratamientos específicos, pero siempre bajo la supervisión de los jueces. A pesar de la aparente efectividad de estos juzgados, resulta evidente que muchos casos relacionados con la drogadicción quedan fuera de estos programas, debido al hecho de que, en muchos casos, la naturaleza de los delitos cometidos impide a sus responsables acojarse a los beneficios de dichos programas. Como medida para la solución de este problema se ha sugerido que los drogadictos puedan acogerse a los juzgados de tratamiento durante la etapa final del cumplimiento de la sentencia, convirtiendo a estos juzgados en auténticos juzgados de reinserción social (reentry courts).

Resulta indiscutible que las áreas a las que se dedican los juzgados de tratamiento son áreas que merecen la atención especial que se les está brindado. No obstante, no podemos perder de vista que existen otras áreas importantes que requieren también de consideración, y especialmente aquellas relacionadas con la reinserción social del exconvicto. La permanencia en la prisión trae consigo efectos psicológicos tanto para el drogadicto como para el que no lo es; por lo mismo, al buscar soluciones para la rehabilitación de la persona, evitando en la medida de lo posible su reincidencia, no podemos limitarnos sólo a los drogadictos, sino que es necesario extender estas soluciones a todos los convictos en general.

La reinserción social no se produce de un momento a otro, sino que es más bien un proceso de varias etapas, en cada una de las cuales el apoyo que se le brinde a la persona será un factor determinante para el éxito de su reinserción. Una buena manera para hacer llegar ese apoyo es la planificación cuidadosa, gradual e individual de la reintegración del delincuente a la comunidad. Ello ha sugerido el establecimiento de un

\footnotetext{
${ }^{2}$ Para mayor información sobre los principios y prácticas del derecho terapéutico puede dirigirse a la página web oficial: http:// www.therapeuticjurisprudence.org, donde encontrara artículos en castellano así como una bibliografía sobre la materia.
}

Revista Española de Investigación Criminólogica

REIC AC-01-04

http://www.criminologia.net

ISSN 1696-9219 
nuevo tipo de juzgados de tratamiento especializado que asumiera estos objetivos, en la forma de un jusgado de reinserción social accesible para todos los convictos (Winick \& Wexler, 2003). Si bien bajo el modelo jurídico-penal vigente en los Estados Unidos, la creación de estos juzgados de reinserción se hace sumamente difíci, la tarea se facilitaría si tuvieramos a nuestro alcance un figura jurídica adecuada, como puede ser la del juez de vigilancia; una figura que, al menos en teoría, ofrece un marco jurídico idóneo para la creación de juzgados de tratamiento especializado que puedan operar como juzgados de reinserción para delincuentes adultos.

Al analizar la situación de los juzgados de reinserción social desde una perspectiva de derecho comparado entre España y Estados Unidos, nos percatamos de la existencia de cierta ironía. Mientras que en Estados Unidos se ha desarrollado una perspectiva interdisciplinaria, la de la therapeutic jurisprudence, que ha elaborado principios psicológicos que pueden ser útiles para los juzgados de reinserción, se carece lamentablemente del marco jurídico necesario y adecuado para que los jueces puedan mantener algun tipo de contacto con el ofensor en etapas posteriores al propio internamiento. Por contra, en España, donde la therapeutic jurisprudence se encuentra aún en una etapa muy prematura, se cuenta ya con un marco jurídico que, en teoría, pudiera servir como modelo para algunas jurisdicciones angloamericanas. Nuestra conclusión se limita al marco jurídico del JVP, sin atenernos al funcionamiento efectivo de esta figura.

En este artículo argumentamos que la estructura del JVP resulta deseable, hasta cierto punto, para algunos sistemas angloamericanos, ofreciendo pautas que pueden ser útiles para la creación de juzgados de reinserción social en estos sistemas. No estamos proponiendo por tanto cambios específicos en el sistema español, sino, al contrario, recurrimos a éste como modelo que puede servir para generar cambios en los sistemas angloamericanos. En nuestra exposición, haremos referencia a los principios de la threapeutic jurisprudence, principios que no se limitan necesariamente a la creación de juzgados de reinserción social y que pueden tener una potencial aplicación en una pluralidad de procesos jurídicos.

\section{El marco jurídico del JVP}

La figura del juez de vigilancia penitenciaria (en adelante, JVP) fue creada con el propósito de salvaguardar los derechos de los internos, con miras a ofrecer un mecanismo de control jurisdiccional de la actividad penitenciaria en contra de la arbitrariedad en las actuaciones del poder ejecutivo. La figura toma como modelo el sistema de jueces de aplicación de condenas existentes en otros países europeos ${ }^{3}$.

En términos generales, al JVP se le atribuye el papel de resolver, en sede judicial, cuantas cuestiones puedan plantearse en el ámbito de la ejecución de las penas privativas de libertad, asumiendo así las funciones que de otra forma corresponderían al tribunal sentenciador. Las funciones que le corresponden al JVP quedan recogidas en el artículo 76 de la Ley Orgánica 1/1979. Entre las funciones que se enumeran en dicho artículo, merecen una especial atención las siguientes:

\footnotetext{
${ }^{3}$ Un ejemplo mas concreto sería la figura del Magistrado de Supervision de Italia Véase Ciappi et al., 2001.

Revista Española de Investigación Criminólogica

REIC AC-01-04

http://www.criminologia.net

ISSN 1696-9219
} 
- Adoptar todas la decisiones necesarias para que los pronunciamientos de las resoluciones en orden a las penas privativas de libertad se lleven a cabo ${ }^{4}$.

- Resolver sobre las propuestas de libertad condicional de los penados y acordar las revocaciones que procedan ${ }^{5}$.

- Resolver, sobre la base de los estudios de los equipos de observación y de tratamiento, y en su caso de la central de observación, los recursos referentes a la clasificación inicial y a progresiones y regresiones de grado ${ }^{6}$.

- Acordar lo que proceda sobre las peticiones o quejas que los internos formulen en relación con el régimen y el tratamiento penitenciario en cuanto afecte a los derechos fundamentales o a los derechos y beneficio penitenciarios de aquellos ${ }^{7}$.

Las funciones del JVP se concentran por tanto en la etapa de aplicación de la condena. No obstante, no podemos perder de vista que uno de los objetivos fundamentos del derecho penal es el de alcanzar una mejor convivencia social. La imposición de una pena restrictiva de libertad, en principio, aspira a conseguir readaptar socialmente al individuo de manera que se consiga evitar que éste cometa nuevas conductas antisociales, lo que a su vez redundaría en una mejor convivencia social.

Con miras a lograr el éxito en su cometido, todo proceso de readaptación social debe comenzar mientras el interno cumpla su condena y sin esperar a que se acerque la fecha de su puesta en libertad, ya que para entonces podría ser demasiado tarde. Partiendo de esta perspectiva, la Ley Orgánica 1/1979 establece que cada interno, durante el cumplimiento de su condena, será objeto de un tratamiento penitenciario individualizado dirigido a su reeducación y reinserción social ${ }^{8}$. No conforme con ésto, la misma ley establece que debera fomentarse la participación activa del penado en la planificación y ejecución de su tratamiento ${ }^{9}$. Puede concluirse entonces que el ordenamiento jurídico español reconoce al penado el derecho a recibir un tratamiento adecuado que propicie su readaptacion social, y un derecho además protegible. Siendo el JVP la persona sobre la que recae la tarea de salvaguardar los derechos de los internos, incluyendo el derecho a un tratamiento adecuado, el sistema atribuye al juez un papel muy activo en el proceso de readaptación del interno.

El tratamiento que reciba el convicto cuenta con la supervisión de equipos cualificados de especialistas. La evolución y progreso en el tratamiento del interno lo que determinará su clasificación, que deberá ser actualizada cada seis meses como máximo. Esta clasificación constituye un requisito indispensable para la concesión de libertad condicional, que a su vez requiere de la aprobación delJVP.

Según dispone el Código Penal ${ }^{10}$, la libertad condicional podrá ser concedida a aquellos convictos que cumplan, entre otros, con los siguientes requisitos:

\footnotetext{
${ }_{5}^{4}$ Ley Orgánica No. 1/1979, General Penitenciaria, art. 76(a)

${ }_{6}^{5}$ Ibid., art. 76(b)

${ }^{6} \mathrm{Ibid}$. , art. $76(\mathrm{~d})$

${ }^{7}$ Ibid., art. 76(e).

${ }^{8}$ Ibid., art. 59.

${ }^{9}$ Ibid., art. 61 .

${ }^{10}$ Ley Organica 10/1995 de 23 de noviembre, del Código Penal, art. 59.

Revista Española de Investigación Criminólogica 
i) se encuentren en tercer grado de tratamiento penitenciario;

ii) hayan cumplido las tres cuartas partes de la condena; hayan observado una buena conducta y exista respecto de los mismos un pronóstico individualizado y favorable de reinserción social emitidos por los expertos que el juez de vigilancia penitenciaria estime convenientes.

Al decretar la libertad condicional, el JVP podra imponer la observancia de una o varias reglas de conducta de las previstas en el articulado del Código Penal. Entre las medidas que puede imponer el JVP podriamos resaltar las siguientes:

- comparecencia personale ante el Juzgado o Tribunal, o servicio de la Administración que éstos señalen, para informar de sus actividades y justificarlas ${ }^{11}$.

- participación en programas formativos, laborales, culturales, de educación vial, sexual y otros similares ${ }^{12}$.

- cumplimiento de los demás deberes que el Juez o Tribunal estime convenientes para la rehabilitación social del penado, previa conformidad de éste y siempre que no atente contra su dignidad como persona ${ }^{13}$.

Así mismo, el JVP podrá decretar que se comparta la custodia del interno con algún familiar. Esta medida implica que el sometido a ella queda sujeto al cuidado y vigilancia del familiar que se designe y que acepte la custodia, quien a su vez la ejercerá en relación con el JVP y sin menoscabo de las actividades escolares o laborales del custodiado $^{14}$.

De concederse la libertad condicional, le corresponderá al centro penitenciario más próximo al lugar donde vaya a residir el convicto la supervisión del funcionamiento de los servicios sociales penitenciarios. A su vez, se elaborará un plan individualizado que establezca el seguimiento de los beneficiarios de la libertad condicional en el cual el JVP podrá incorporar "reglas de conducta" 15 . Según establecen las disposiciones del Código Penal que enumeran estas reglas, el JVP cuenta con una amplia capacidad para supervisar el cumplimiento de las mismas, pudiendo además decretar las reglas que estime conveniente, siempre que se salvaguarde el derecho del penado a la dignidad y que éste preste su consentimiento. Esto último representa una cláusula abierta que puede llevar a la imposición de medidas de seguridad más adecuadas.

\section{Elementos principales de la figura del JVP}

Son varios los aspectos de la legislación española que la convierten en un posible modelo a seguir para la creación de juzgados de reinserción en los sistemas angloamericanos. En esta sección analizaremoslos aquellos elementos de la figura del JVP que pueden resultar provechosos para el establecimiento de tribunales de reinserción social en los sistemas angloamericanos.

${ }^{11}$ Ibid., art. 83(3).

${ }^{12} \mathrm{Ibid}$., art 83(4).

${ }^{13} \mathrm{Ibid}$., art. 83(5).

${ }_{15}^{14}$ Ibid., art. 105(e).

${ }^{15}$ Ibid., art. 85.

Revista Española de Investigación Criminólogica

REIC AC-01-04

http://www.criminologia.net

ISSN 1696-9219 
1. La autorización para la aprobación de la libertad condicional corresponde a un solo juez, en vez de recaer sobre un órgano colegiado.

El éxito de los juzgados para el tratamiento de la drogadicción se debe en gran medida a la relación interpersonal que se desarrolla entre el juez y el ofensor (Winick \& Wexler, 2003). El desarrollo de una buena relación interpersonal entre el ofensor y el juez coloca a este último en una excelente posición para convertirse en un agente motivador en la rehabilitación del ofensor. En este sentido, un sistema que conste de un solo juez resulta más aconsejable que uno en el que las decisiones recaigan sobre varias personas.

En el sistema de parole existente en los Estados Unidos hasta hace poco, la libertad condicional se concedía por un órgano administrativo colegiado (parole board). El hecho de que estos órganos estuvieran integrados por varias personas, a veces cambiantes, representaba un claro impedimento para del desarrollo de una relación interpersonal, y por tanto de una relación efectiva de trabajo, con el convicto.

\section{El JVP ejerce un rol activo desde el momento del encarcelamiento del convicto.}

Este aspecto presenta dos corolorarios. En primer lugar, el papel del órgano judicial comienza mucho antes de que el ofensor pueda acceder a los beneficios de la libertad condicional. De este modo, el juez puede tomar un papel activo en el progreso del convicto desde una etapa temprana del cumplimiento de la pena El juez puede contribuir desde temprano en la aparición en la conciencia del convicto de un compromiso como miembro de la sociedad en la que vive, en el marco del respeto a la ley.

Esta caraterística del modelo del JVP contrasta con el sistema de libertad condicional previamente existente en los Estados Unidos, en el que el órgano responsable de conceder la libertad condicional, el parole board, sólo establecía contacto con el penado una vez que éste había cumplido una parte sustantiva de la condena, privándose así al sistema de un importante potencial en términos de la rehabilitación social de la persona.

En segundo lugar, la función judicial de corrección y reinserción recae sobre un juez diferente a aquel que dicta la sentencia. Algunos comentaristas se han pronunciado a favor de una posición diferente para los posibles juzgados de resinserción en los Estados Unidos, sugiriendo que el juez sentenciador debería ser el mismo que actuará luego en el proceso de reinserción (Petersilia, 2003). Ciertamente, esta combinación de roles es posible y en teoría podría producir buenos resultados. No obstante, en la medida en que el convicto puede guardar cierta descofianza o resentimiento hacia el juez sentenciador, resulta recomendable que estos roles sean ejercidos por figuras independientes, en línea con el ordenamiento español. Al igual que en el sistema del JVP, en el marco jurídico que proponemos el juez de reinserción debe ser una una persona diferente al juez sentenciador, permitiendo así que los convictos puedan verlo como una persona con interés genuino en la protección de sus derechos y en su rehabilitación.

3. La libertad condicional no se concede automáticamente cuando se cumple con determinada parte de la condena, ni tampoco depende de la total discreción del JVP, sino que está condicionada al progreso del prisionero en las distintas etapas de la condena.

Revista Española de Investigación Criminólogica

REIC AC-01-04

http://www.criminologia.net

ISSN 1696-9219 
Este tipo de sistema posee varios aspectos positivos. Para empezar, reduce la arbitrariedad que caracteriza a los sistemas discrecionales, como es el caso tradicional de los sistemas de libertad bajo palabra jurisdicciones todavía vigente en algunos países. Asimismo, el sistema español reduce la arbitrariedad, permitiendo al juez sólo una discreción limitada en el momento de la concesión de la libertad condicional. Esta caracererítica contrasta con el antiguo sistema de discretionary parole de los Estados Unidos, donde.la excesiva discreción reconocida a los miembros de la junta de librtead condicional (parole board) limitada el efecto terapéutico de su labor. La limitación de la discreción del JVP puede hacer disminuir la arbitrariedad y los efectos antiterapéuticos relacionados con ésta, al tiempo que mantiene la motivación en los prisioneros, en la medida en que el cumplimiento de los requisitos de la libertad condicional dependerá en gran medida de su propia voluntad y el esfuerzo.

Por otra parte, aunque al JVP se le reconozca una discreción sólo limitada, ésta es suficiente para que éste pueda suspender efectivamente el cumplimiento de la condena. Esta característica de nuevo se convierte en una fuente de motivación para el penado, que puede percibir cómo los prgresos en su conducta pueden afectar a la concesión de la libertad condicional. En el sistema de los Estados Unidos, por el contrario, la libertad condicional se concede en algunos casos en el momento mismo en el que se dicta la sentencia, por lo que se pierde su potencial como instrumento motivador y rehabilitante.

4. La ley que define la figura del JVP permite expresamente la imposición de condiciones como parte del proceso de libertad condicional, incluyendo entre estas la celebración de audiencias de seguimiento.

La autorización para establecer varias condiciones de libertad favorece la posibilidad de establecer un dialogo entre el ofensor y el juez, permitiendo que estas condiciones puedan conceptualizarse más como un acuerdo entre las dos partes que como una orden judicial unilateral. Independendientemente de lo que sucede en la práctica, el hecho de que el reo conceptualize las condiciones como un acuerdo puede promover en éste un compromiso con el cumplimiento de estas condiciones. A la vista del ejemplo de los juzgados de tratamiento de la drogadicción, en el que la continua supervisión judicial es un factor crucial para la rehabilitación de los penados, podría concluirse igualmente que tanto el desarrollo de una relación entre el juez y el ofensor como la continuidad de ésta una vez alcanzada la libertad son elementos importantes importante en el contexto de la reinserción.

\section{Algunos principios de la therapeutic jurisprudence que pudieran combinarse con el modelo del JVP}

Evidentemente, un marco jurídico adecuado es sólo una primer paso en la creación de juzgados de reinserción social. Para lograr el cumplimiento del os objetivos de estos juzgados, se hará necesario combinar este marco jurídico con la aplicación de los principios de la therapeutic jurisprudence.

La therapeutic jurisprudence pretende que los jueces que reconozcan su papel como agentes terapéuticos, y que además estén dispuestos a ejercer su este papel con una

Revista Española de Investigación Criminólogica

REIC AC-01-04

http://www.criminologia.net

ISSN 1696-9219 
sensibilidad humana. Aquellos jueces que acogen esta visión pueden recurrir a los principios de la therapeutic jurisprudence ${ }^{16}$.

La teoría interdisciplinaria de la therapeutic jurisprudence es el producto de un esfuerzo constante por incorporar conocimientos de las ciencias del comportamiento en la práctica legal y judicial. En esta sección discutiremos brevemente algunos de los principales principios de la therapeutic jurisprudence y examinaremos cómo estos principios podrían combinarse con un marco jurídico similar al del JVP, de manera que puedan servir de modelo para la creación de los juzgados de reinserción en los sistemas angloamericanos.

\section{A. Comunicación y aptitudes interpersonales.}

Varios estudios recientes sobre el efecto de la práctica terapéutica han mostrado resultados positivos. Curiosamente, estos estudios demuestran que en la mayoría de los casos el éxito de la terapia no depende tanto de técnicas especificas, sino que los mejores resultados se obtienen cuando se atiende a factores particulares del convicto. Un factor importante en el éxito de esta terapia es la relación que se desarrolle con el convicto, así como su capacidad para generar esperanza y expectativa en éste ${ }^{17}$.Por lo tanto, desde el punto de vista de un juez con interés en convertirse en un agente de cambio de comportamiento, la mayor parte del trabajo terapéutico debe ir dirigida a lograr una participación significativa del convicto, así como a desarrollar una relación estable con el victo que permaita generar en éste un nivel crítico de esperanza y de expectativa del cambio.

Sólo podrá establecerse una relación interpersonal estable entre el juez y el convicto cuando el primero procura dirigirse al segundo con dignidad y respeto, $r$, reconociéndole a la persona un espacio para dejar oír su voz, así como la oportunidad de contar su historia y de generar el sentimiento de que su historia será tomada en serio. Si el juez está pendiente del convicto, le explica los procedimientos, y le ofrece una oportunidad para expresarse y explicar las decisiones tomadas en el pasado y las razones que le indujeron a tomarlas, la persona será más receptiva a aceptar las órdenes del juez y a cumplirlas efectivas (Winick \& Wexler, 2003).

Un marco jurídico que ofrezca la posibilidad para que el juez interactúe con el convicto, como en teoría sucedere con el JVP, puede facilitarfel desarrollo de este tipo de

\footnotetext{
${ }^{16}$ Recientemente muchos de estos principios han sido expuestos en el libro Judging in a Therapeutic Key: Therapeutic Jurisprudence and the Court editado por Bruce J Winick y David B Wexler y publicado por Carolina Academic Press (2003). Para una traducción en castellano de la introducción del libro, puede dirigirse a la sección de artículos en castellano de la página web http://www.therapeuticjurisprudence.org.

${ }^{17}$ Estudios recientes sobre los resultados de las prácticas terapéuticas sugieren que los factores relacionados con el convicto y su contexto social, tales como sus características personas y situaciones particulares, son los más importantes para promover resultados positivos (llegando alcanzar un $40 \%$ de cambios positivos). De igual forma, estos estudios sugieren que los modelos y técnicas terapeuticas específicas solo promueven un $15 \%$ de éxito en los cambios. Otro $15 \%$ de cambio positivo se atribuye a los factores de esperanza y expectativa. Por lo demás, resulta interesante que una gran porción de los resultados positivos $(30 \%$ de la contribución al cambio) se relacionan con factores de relación entre el convicto y el agente de cambio, quien en determinados contextos puede ser el juez (Winick \& Wexler, 2003).
}

Revista Española de Investigación Criminólogica REIC AC-01-04 http://www.criminologia.net

ISSN 1696-9219 
relación entre el juez y el convicto. En la medida en que el JVP forma parte de las decisiones en torno a la clasificación de los internos, y siendo necesaria una clasificación de tercer grado para obtener la libertad condicional, el juez cuenta con una gran oportunidad para ir desarrollando algún tipo de relación interpersonal con el convicto durante toda la etapa anterior a la concesión de la libertad condicional. Asimismo, una vez que se concede la libertad condicional, el juez podrá requerir la celebración de audiencias de seguimiento como parte de las reglas de conducta que el convicto debe respetar. La concepción del JVP como el responsable de velar por los derechos de los internos y como una figura separada de la del juez sentenciador, ofrece nuevas oportunidades para facilitar el desarrollo de una relación efectiva con los internos que promueva su rehabilitación.

B. Reducción de la reincidencia a través del desarrollo de aptitudes en el convicto para la solución de sus problemas.

A través de los años los estudios sobre la rehabilitación de los convictos no han mostrado resultados muy alentadores. Sin embargo, estudios más recientes han sacado a la luz un cierto éxito de los métodos de tratamiento enfocados en el desarrollo de aptitudes en el convicto para promover la solución razonada de sus problemas. Estas técnicas, generalmente conocidas como "técnicas de comportamiento cognoscitivo", fomenta en aquellos internos impulsivos y con poco autocontrol el reconocimiento de la cadena general de eventos que generalmente les incita a ejercer conductas punibles, así el reconocimiento de aquellas situaciones que pudieran ser consideradas "situaciones de alto riesgo" (p. ej., socializar con determinadas personas, ir a la discoteca los viernes por la noche, etc). En su aplicación práctica, esta técnica pretende que el convicto analice y considere actividades y conductas alternativas, de manera que, en la medida de lo posible, evite encontrarse en estas situaciones de riesgo (p. ej., no salir con determinada persona, ir al cine en vez de ir a la discoteca). En ciertas ocasiones se ofrece a los convictos cursos de "rehabilitación y razonamiento", así como tareas para formular un "plan para la prevención de la reincidencia".

Usando los principios de la therapeutic jurisprudence, el modelo del JVP podría servir para promover efectivamente en el convicto el desarrollo de estas aptitudes de comportamiento cognoscitivo. Por ejemplo, el JVP, en el momento de evaluar el expediente del convicto con miras a la concesión de la libertad condicional, podrá proponer a éste que prepare, con la ayuda del criminólogo, el abogado o un equipo de profesionales, un plan de prevención de la reincidencia, que podrá sera usado como base para discutir la aprobación de su solicitud de libertad condicional.

Asimismo, sería ideal que se ofreciera un curso de "razonamiento y rehabilitación" en la prisión. De realizarse estos cursos, si el JVP acepta la presentación de planes de prevención de la reincidencia por parte los solicitantes de libertad condicional, los convictos podrían beneficiarse de haber realizado estos cursos en la prisión, por más que técnicamente no estuvieron obligados a seguirlos.

De seguir este tipo de patrón, el JVP estaria fomentando en el ofensor el desarrollo de aptitutes importantes que podrán ayudarle en su readaptación a la sociedad. Y, además, si el convicto reconoce cuáles son sus situaciones de alto riesgo y propone métodos de acción alternativa para evitar tales situaciones (no ir a la discoteca los

Revista Española de Investigación Criminólogica

REIC AC-01-04

http://www.criminologia.net

ISSN 1696-9219 
viernes), sería básicamente éste, el mismo convicto, el que estaría proponiendo sus propias condiciones de libertad. De este modo, la persona comprenderá mejor el contenido de las medidas impuestas, así como la razón de las mismas. En tal caso, el convicto no tenderá a percibir estas medidas como condiciones impuestas injustamente por un agente jurídico externo, razón por la cual presentará probablemente una mayor disposición para cumplirlas (Wexler, 2001) ${ }^{18}$.

\section{La promoción del compromiso del convicto con el cumplimiento de las condiciones de libertad condicional.}

El componente de comportamiento cognoscitivo de la therapeutic jurisprudence se encuentra íntimamente relacionado con los principios desarrollados por esta disciplinaen relación con el cumplimiento de las condiciones de libertad condicional, un aspecto donde también el modelo del JVP puede ofrecer un marco adecuado para promover el éxito de la rehabilitación del penado. El JVP debe asegurarse que el convicto al que se le conceda el beneficio de la libertad condicional internalice el compromiso de acatar las condiciones de libertad impuestas. Ciertamente, mientras más confianza tenga el juez en el cumplimiento de las condiciones con mayor probabilidad aprobará la libertad condicional.

La therapeutic jurisprudence plantea ciertos principios que los jueces pueden aplicar para desarrollar y acrecentar en el convicto la voluntad de cumplir con las condiciones de la libertad condicional (Winick \& Wexler, 2003). Será más fácil alcanzr un compromiso de cumplimiento en el convicto si, por ejemplo, el juez utiliza un lenguaje claro (en vez de un lenguaje técnico) cuando se dirige a éste, y cuando consigue conceptuar la libertad condicional no como una orden judicial unilateral, sino como un "contrato de comportamiento" que ofrezca al convicto la oportunidad de participar en el desarrollo de su plan (p. ej., "Te aprobaré a libertad condicional si te comprometes en hacer X, Y y Z" o "Me comprometo en hacer X, Y y Z si me aprueba la libertad condicional"), aceptando la presencia de familiares en las audiencias de seguimiento y utilizando éstas como un foro donde el convicto pueda hacer un compromiso público para cumplir con estas condiciones.

Es importante señalar nuevamente que el cumplimiento de las condiciones de la libertad provisional pueden ser más efectivos en la medida en que ésta no sea concedida automáticamente, permitiendo que le juez conceptualice la concesión de la libertad como un contrato de comportamiento cuyos términos estarán sujetos a discusión en la vistas judiciales posteriores (Winick \& Wexler, 2003).

\section{Enfrentando los riesgos y construyendo vidas mejores.}

Cuando el JVP considere la aprobación de la libertad condicional deberá conocer cuáles son los riesgos de reincidencia del ofensor: es decir, el juez debe convertise, en alguna medida, en un agente de manejo de los riesgos. Para alcanzar este objetivo, todo JVP que tenga disposición parsa la rehabilitación deberá prestar una especial atención a

\footnotetext{
${ }^{18}$ Para una versión en castellano del artículo citado, puede dirigirse a la sección de artículos en castellano de la siguiente página: http:// www.therapeuticjurisprudence.org. La versión original del artículo puede encontrarse en la seccion de publicaciones de la siguiente página: http://aja.ncsc.dni.us.

Revista Española de Investigación Criminólogica
} 
los factores dinámicos de riesgo, tales como el uso y abuso de alcohol y otras drogas. Las herramientas para enfrentar el riesgo pueden servir no sólo para predecir los riesgos futuros, sino también para motivar al ofensor a que modifique estos factores de riesgo. Por medio de estas herramientas, se estaría ofreciendo al convicto la oportunidad de dar tomar un paso significativo hacia la rehabilitación, al tiempo que así como para maximizar las oportunidades de labrarse un porvenir en libertad (Winick \& Wexler, 2003).

El JVP puede ejercer un papel importante como agente de manejo de riesgo cuando analiza las progresiones y retrocesos en la clasificación del interno, así como en las vistas de seguimiento que se celebren como parte de las medidas de seguridad asocidas a la concesión de la libertad condicional. La legislación española que establece la figura del juez de vigilancia le reconoce explícitamente a éste la capacidadde ordenar la comparecencia del ofensor ante el juzgado para que éste informe sobre sus actividades. Asimismo, como vimos anteriormente, la la Ley Orgánica 1/1979 no ofrece una lista taxativa de medidas de seguridad, pudiendo el JVP establecer cualquier otra medida que considere necesaria, siempre que se respete el derecho a la dignidad del interno. Desde la perspectiva de la therapeutic jurisprudence, este tipo de audiencias ofrece una buena oportunidad para alcanzar resultados terapéuticos.

Al mismo tiempo, es importante que tanto a los convictos cercanos a ser liberados, como a aquellos que ya se han acogido a la libertad condicional, se les ofrezca la oportunidad de participar en programas sociales o educativos, así como en programas de formación profesional.

En las vistas de seguimiento, el JVP no tiene por qué limitarse necesariamente a velar el cumplimiento de las condiciones de libertad provisional, sino que también puede aprovechar estas vistas para supervisar el correcto funcionamiento de los servicios sociales (Winick \& Wexler, 2003). Ello resulta especialmente importante desde la perspectiva del modelo de rehabilitación para una "vida mejor", un modelo mucho más positivo que el de mero manejo de riesgo o prevención de futuros crímenes. Este modelo sugiere que "la manera en que se reduce la reincidencia es dándole a los individuos las herramientas necesarias que los guíen hacia un mejor estilo de vida, en vez de simplemente enseñarles a minimizar la probabilidad de volver a ser encarcelados"(Ward \& Stewart, 2003).

\section{E. El mantenimiento del comportamiento social mediante el reconocimiento judicial.}

En un recente estudio, Maruna ha puesto de manifiesto el importante papel que juega el reconocimiento y reforzamiento de los logros alcanzados por los convictos que se enfrentan a un en proceso de replanteamiento de sus vidas, desistiendo de la conducta criminal (Maruna, 2001). Este desistimiento es un proceso contínuo, que podrá alcanzarse más fácilmente cuanto mayor sea el reconocimiento por parte de figuras relevantes dentro de esfera social del convicto y de los responsables de los servicios sociales.

A título de ejemplo, los juzgados para el tratamiento de drogadición existentes en los Estados Unidos llevan a cabo actividades de "reconocimiento del cambio" durante las vistas de seguimiento, así como las denominadas "ceremonias de graduación”, que se celebran cuando el convicto culmina con éxito un programa de tratamiento y se retiran

Revista Española de Investigación Criminólogica

REIC AC-01-04

http://www.criminologia.net

ISSN 1696-9219 
los cargos penales. Es común que en estas ceremonias asistan los familiares y amistades del convicto, que junto al juez aplauden los esfuerzos realizados por el éste para lograr el éxito de su rehabilitación.

El éxito del modelo de "reconocimiento de cambio" de los juzgados de tratamiento de la drogadicción hace deseable su adaptación a otros escenarios, tales como las audiencias de seguimiento, o en el momento en que se culmine con éxito el periodo de libertad condicional. Lo importante es que estas ceremonias sean terapéuticas por sí mimas, y que no sean meramente "rituales" (Winick \& Wexler, 2003).

Por lo general, se recomienda la celebración de audiencias de seguimiento cuando existe el temor de que el convicto puede incumplirlas reglas de conducta de la libertad condicional, existiendo la posibilidad de la imposición de sanciones o incluso de la revocación total de esta libertad. No obstante, como hemos señalado, pueden existir otras razones importantes por las cuales se deben llevar a cabo tales audiencias, incluyendo la supervisión del correcto funcionamiento de los servicios sociales. Asimismo, en esta sección hemos apuntado a otra razón importante para la celebración de estas vistas: los aparentes resultados terapéuticos de las ceremonias y reconocimientos judiciales que pueden llevar a cabo en dichas audiencias. Todo ello hace que las vistas de seguimiento autorizadas la legislación española como medida de conducta resvitan importancia aún cuando no existan problemas significativos con el funcionamiento de los servicios sociales o con la conducta del convicto.

\section{Conclusión}

La figura del JVP resulta desconocida en el mundo jurídico angloamericano. Sin embargo, esta figura se adaptaría muy bien a los intereses y esfuerzos por remediar la lamentable situación ante la cual se enfrentan en la actualidad los prisioneros en el momento de su puesta en libertad.

Es necesario buscar nuevas alternativas que propicien la rehabilitación del prisionero. El éxito de un sistema de justicia penal no debe medirse segun los delincuentes que consiga encerrar, sino más bien debe medirse según aquellos que logra rehabilitar, convirtiéndolos así en un individuo comprometido con la sociedad, y no en un peligro para ésta. Dentro de este proceso de rehabilitación del delincuente, la rehabilitación social juega un papel fundamental. A juzgar por el éxito de los juzgados de tratamiento, la creación de juzgados de reinserción social puede ser una buena alternativa para los sistemas angloamericanos.

Sin embargo, a pesar de ser una cada vez más aceptada, la creación de dichos juzgados se enfrenta con grandes dificultades en la práctica. La razón reside en el hecho de que, si bien contamos con los elementos necesarios para el establecimiento de dichos juzgados, carecemos todavía en nuestros países del marco jurídico necesario para su creación y puesta en funcionamiento.

En este artículo hemos argumentado que la legislación española referente al JVP puede servir como modelo para los sistemas angloamericanos que carezcan de un marco jurídico adecuado para la creación de los juzgados de reinserción. La creación de estos juzgados debería combinar este tipo de marco jurídico con los principios de la therapeutic jurisprudence anteriormente expuestos.

Revista Española de Investigación Criminólogica

REIC AC-01-04

http://www.criminologia.net

ISSN 1696-9219 
Con todo, las nociones de la therapeutic jurisprudence no se limitan a la enumeración de principios para la creación de juzgados de reinserción, sino pueden ser usados por todo aquel que sienta la inquietud de convertirse en un agente de cambio. Desde nuestra perspectiva, el análisis de los principios de la therapeutic jurisprudence en éste y otros contextos puede propiciar un diálogo fructífero en el marco del derecho comparado.

\section{BIBLIOGRAFÍA}

Ciappi Silvio, Wexler, David, Agrait, Fernando y Giovanni Traverso 2001. Therapeutic Jurisprudence. Alcune considerazioni su di una concezione postliberale del diritto e della pena. ReSSEgnA It. Di CRIMINOLOGIA. Italia. 12(3/4): 355-373.

Maruna, Shadd. 2001. Making Good: How Ex-convicts Reform and Rebuild Their Lives. Washington, D.C.: American Psychological Association Books.

Petersilia, Joan 2003. When Prisioners Come Home. New York: Oxford University Press.

Ward, T \& C. A. Stewart. 2003. Criminogenic Needs and Human Needs: A Theoretical Model. Psychology, Crime \& Law.: 234-345.

Wexler, David 2001. Robes and Rehabilitation: How Judges Can Help Offenders "Make Good”. COURT REVIEW 38(1): 18-23.

Para la traducción al castellano de este artículo, véase la sección de artículos en castellano en: http://www.therapeuticjurisprudence.org

Winick, Bruce y David Wexler. 2003. Judging in a Therapeutic Key: Therapeutic Jurisprudence and the Courts. North Carolina: Carolina Academic Press.

David Wexler ha escrito seis libros, el más reciente de los cuales es Judging in a Therapeutic Key: Therapeutic Jurisprudence and the Courts (2003)El autor ha fungido como Fulbright Senior Specialist, ofreciendo conferencias sobre therapeutic jurisprudence en Nueva Zelanda y Australia. Es además consultor del Instituto Judicial Nacional de Canadá en estas cuestiones. Para información adicional sobre el autor puede consultar la sección "About the director" en la siguiente página web: http://www.law.arizona.edu/depts/upr-intj/

Jeanine Calderón es estudiante de la Escuela de Derecho de la Universidad de Puerto Rico.

Revista Española de Investigación Criminólogica

REIC AC-01-04

http://www.criminologia.net

ISSN 1696-9219

https://doi.org/10.46381/reic.v2i0.14 